\title{
Marginalités et fantaisies linguistiques chez Boris Vian.
}

\begin{abstract}
:
In this analysis we are interested in certain stylistic and rhetorical aspects in three Birus Vian novels texts. We wonder about the various processes used by the author for his verbal-linguistic creations. Our goal is to identify the marginal feature of Vian's work and to understand its mechanism.
\end{abstract}

Keywords: Marginalities, creation, style, processes, linguistics.

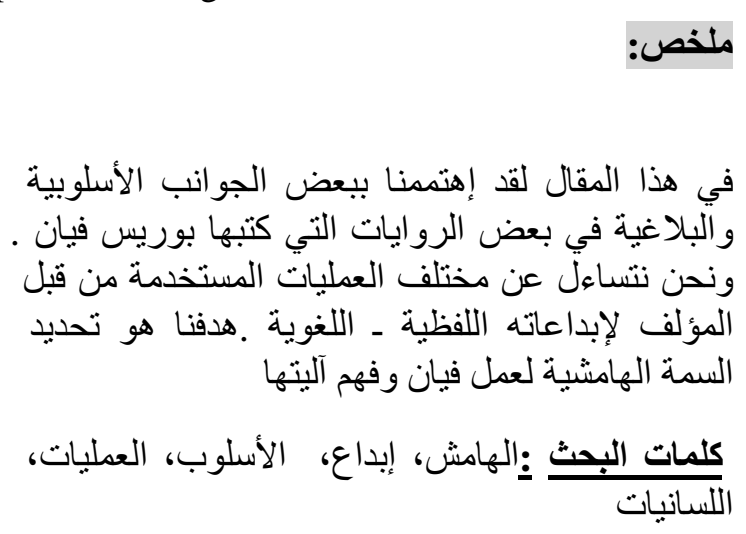

\section{Khebbab khaoula}

Département de lettres et de la langue Française

Université des frères Mentouri

Constantine, Algérie.

\section{Introduction}

La notion de marginalité renferme tout ce qui est en marge de la normalité, tout ce qui diffère de ce que l'on connaît, tout ce qui ne s'applique pas aux normes préconçues et établies.

Marginalité dans le fait littéraire désigne "...tous les aspects rares ou inhabituels de la vie littéraire peuvent être qualifiés de marginaux : les poètes lyriques au XVIIIe $s$, les femmes écrivains au XIXe s, les tragédiens classiquesauXXe. Toutefois l'expression "marginalité 
littéraire" a pris un sens plus précis dans la critique depuis les années 1960. Elle désigne généralement les instances, les producteurs, les organisations, les pratiques et/ou les corpus non reconnus pour légitimes par et au sein de l'institution littéraire $»^{1}$.

Bien qu'il ait côtoyé les oulipiens, qu'il ait été l'ami de Raymond Queneau, qu'il ait vécu durant les années surréalistes, l'auteur de l'Herbe rouge s'est forgé un style qui lui est bien propre, touche-à-tout et poussant ses idées jusqu'à leur paroxysme fictif et à l'extrême dans ses textes, reculant les limites de la langue et violentant au besoin ses normes, usant de plus de 73 pseudos, Vian est considéré comme un marginal par son style et l'ensemble de son œuvre inclassable.

Dans l'une de ses lettres ${ }^{2}$ à sa femme Ursula en 1951, Vian écrit sa déception et son atterrement face à l'incompréhension de ses lecteurs qui n'arrivent pas à le cerner ainsi que le trait humoristique de son œuvre. Marginal institutionnel, Boris Vian a dû attendre sa mort pour être connu et accepté par le grand public. Ses œuvres sont traduites dans le monde entier et dans différentes langues.

Le but de cette analyse stylistique est de démontrer la manière dont le lexique utilisé par Boris Vian diffère du langage commun usité. Ce qui nous amène à formuler la problématique suivante : par quels moyens stylistiques et rhétoriques l'auteur arrive à créer cet univers -utopique- et comment cette création et ce langage servent le dessein singulier et marginal du romancier?

"Il y a des moments où je me demande si je ne suis pas en train de jouer avec les mots (...). Et si les mots étaient faits pour cela ?» ${ }^{3}$

La plus grande marque d'originalité de Vian demeure son verbe : des mots qui font violence aux normes de la langue, des créations verbauxludiques, des néologismes ${ }^{4}$; Vian ne manque pas de fantaisie quand il s'agit de son outil de travail qu'est la langue.

L’originalité du vocabulaire est multiple. Boris Vian recourt à différents types de néologismes dans le but d'enrichir son œuvre et de lui donner un trait singulier.

\section{Dérivés / dérivation}

La dérivation ${ }^{5}$ dont use Boris dans ses textes, se présente comme un processus de transformation plutôt qu'un processus de création. Rebelle 
des mots, l'auteur enfreint les lois de la grammaire et ses règles traditionnelles. Sa méthode consiste à transgresser toute forme de préfixation et de suffixation habituelles. Vian utilise un préfixe ou un suffixe totalement différent de celui auquel s'attend le lecteur. C'est le cas par exemple de «bidistillée », préfixé par bi- qui représente le dualisme et du participe passé distillé, ce néologisme signifie: doublement distillé voire raffiner le parfum de Chloé.

"Quel parfum avez-vous ? dit-il. Chloé se parfume à l'essence d'orchidée bidistillée. "

Certaines suffixations portent en elles des connotations péjoratives, comme dans l'exemple de la construction du mot vivoir, boudoir, dortoir etc., Boris Vian crée le terme «prioir» (p.123), un renouvellement de «prie-Dieu ». Dérivé de prier, ce mot signifie un endroit où l'on s'agenouille pour faire la prière.

La péjoration du mot prioir vient de la vision satirique et défavorable du personnage principal vis à vis de la religion comme le souligne bien ce passage :

" «Pourquoi l'avez-vous fait mourir ? demanda Colin.

- Oh !... Dit Jésus. N’insistez pas. »

Il chercha une position plus commode sur ses clous.

"Elle était si douce, dit Colin. Jamais elle n’a fait le mal, ni en pensée, ni en action.

- Ça n’a aucun rapport avec la religion », marmonna Jésus en bâillant. " ${ }^{7}$

La liberté linguistique de l'auteur, affecte tout aussi les verbes, de simples substantifs, Boris Vian donne naissance d'une manière ludique à des verbes en leur ajoutant une particule verbale qu'est la terminaison.

Du nom psychiatre, dérive le verbe «psychiatrer »(p.44), de même pour docteur qui donne le verbe «doctoriser ».(p.178)

\section{2- Mots-valises $^{8}$}

La création vianesque est indépendante, rebelle et n'obéit qu'à une seule règle, celle de son auteur. Boris Vian, marche sur les pas de ses ancêtres littéraires dont Jarry et Rabelais mais en ce qui concerne les mots- valises, Lewis Carroll demeure sa seule et unique référence lexicale.

Gilbert Pestureau a évoqué cette influence anglo-saxonne carrollienne dans ce passage : 
"Mais il (Lewis Carroll) en a donné clairement la théorie et des exemples pratiques très nets et très fréquents. Boris, grand lecteur de Snark, n'a pas pu ne pas en être frappé $)^{9}$.

Les trouvailles lexicales de l'auteur touchent plutôt l'ensemble composant la phrase. Avec le verbe comme c'est le cas de "blairnifler » (p.47) construit de blair et de renifler, ce verbe connoterait une idée de voyance, de prédiction olfactive. Nous notons aussi l'adverbe «hypocritégalement», signifiant, comme son nom l'indique, d'une manière également hypocrite. L'auteur s'attaque en outre à l'adjectif qui n'échappe nullement à sa fantaisie, nous relevons l'exemple suivant : « tertreux »(p.110), néologisme construit à priori avec l'adjectif terne et tartreux et qui signifierait terne et sans éclat.

Il y a tout un calendrier de néologismes qui marque avec force les pages de L'arrache-cour. Certains critiques littéraires pensent que Vian pourrait avoir emprunté cette forme de création lexicale à l'auteur russe Gogol.

Janvril (p.125) : janvier + avril / févruin (p. 128) : février + juin / marillet (p.190): mars + juillet / Avroût (p.131) : avril + août / Juinout (p.122). : juin + août / juinet (p.119) : Juin + juillet / juillembre (p.149) : juillet + septembre / octembre (p.161) : octobre + septembre / novembre / décembre/ novrier (p.177) : novembre + février / déçars (p.186): décembres + mars.

L'auteur combine les noms des mois et en crée avec ces combinaisons d'autres désignations, ces dernières soulignent la perturbation de l'écoulement du temps dans la narration.

Cette particularité chronologique n'a lieu que dans la seconde partie du roman L'arrache-cœur et plus précisément à partir du chapitre XII (13 juinet.), cette datation chaotique pourrait se rapporter à priori à la fin de la construction du bateau d'Angel et de son départ. Ce dernier quitte sa famille sur une barque qu'il a construite lui-même.

Le changement temporel n'affecte pas seulement les mois, il touche tout aussi les dates, voire même le nombre classique des jours n'est point conservé. A titre d'exemple :

55 janvril. (p. 125) 107 avroût. (p.133) 348 juillembre. (p. 150)

De plus, dans le monde du possible vianesque, un piano est un mécanisme à liqueurs, un pianocktail (p. 32). Ce mot-valise, est 
composé par télescopage de deux mots étrangers ; piano est l'abréviation d'origine italienne Piano-forte et de l'anglicisme américain cocktail. Cette fantaisie linguistique représente une machine qui permet de fournir la musique et les boissons en même temps. Le principe étant que pour chaque mélodie jouée, le musicien aura un cocktail qui rappellera les impressions ressenties lors de l'écoute du morceau. Soulignons que ce néologisme surréaliste est communément connu comme une version revisitée de l'orgue des saveurs de l'abbé Poncelet dans l'œuvre de Huysman ${ }^{10}$ À rebours.

Enfin, nous conclurons avec le prénom Jacquemort, construit à partir du prénom Jacques (dérivé de Jacob) et du substantif mort, il est l'un des personnages principaux de l'œuvre de Boris Vian. C'est avec lui et en même temps que lui qu'on découvre l'univers de ce roman. Le psychiatre est intérieurement vide et il a besoin de sentiments et d'expériences pour se remplir. C'est cette quête d'émotions qui l'amène au village où a lieu l'intrigue.

\section{3- Mots-composés}

D'une simple juxtaposition lexicale, Vian enrichit le lexique de ses textes avec cette forme de création qui se manifeste dans l'ensemble de notre corpus.

En effet, L'arrache-cœur illustre bien la définition de ce procédé. Composé d'un verbe et d'un nom, cette trouvaille lexicale représente le titre du dernier roman de Boris Vian, mais aussi le nom de l'arme qu'utilise Alise, l'une des protagonistes du roman L'écume des jours, pour tuer l'écrivain Jean-Sol Partre.

Cette arme est peu décrite dans L'écume des jours et n'est jamais citée dans le roman éponyme. On sait que cet arrache-cour est assez petit pour rentrer dans un sac, qu'il a deux branches et qu'il est brillant, qu'on peut le plier et que cet instrument sert, comme son nom l'indique, à arrache les cœurs des poitrines.

On retrouve d'autres exemples de mots composés mais avec une construction différente: nom + nom. Pour illustrer ce modèle de construction nous soulignons dans les textes «Fouille-pétrin» (p.102) ce néologisme signifie dans ce texte, une forme d'herbe comestible qui se mâche et qui rend son consommateur courageux.

\section{4- Autres procédés de création}

\section{4-1 Néosémie ${ }^{11}$ : un mot, d'autres sens}

Autre particularité de la création lexicale vianesque, c’est la néosémie qui consiste à donner au mot une acception nouvelle que celle qu'il 
possède déjà. Le mot dépend de ce qui l'entoure, Vian s'amuse à donner à son vocabulaire d'autres emplois. Dans l'expression : "Il y eut du serrage de mains et Chick en profita pour filer avec Alise. $"{ }^{12}$ L'usage de serrage de main est inapproprié dans la langue usuelle, il s’agit plutôt d'une poignée de main.

De même pour le verbe "comporter » qui signifie dans le dictionnaire : inclure (quelque chose) par nature ou être constitué de. Chez l'auteur de L'Arrache-cœur, «comporter » désignerait porter en plus.

"Elle (Alise) comportait, en outre, un foulard de soie vert vif et des cheveux blonds extraordinairement touffus, encadrant son visage d'une masse frisée serré. ${ }^{13}$

Dans le domaine ecclésiastique, la fantaisie de Vian atteint son paroxysme. "Bedon »(p.105), n'est plus la définition d'un ventre rebondi, il représente tout comme la chevêche, un religieux. Se passant de bedeau, l'auteur lui préfère satiriquement bedon ${ }^{14}$

Par ces procédés lexicaux, Boris Vian tend à faire en sorte que le mot ne soit pas limité à un sens premier ou second, il lui donne une liberté d'emploi, de significations et différentes utilisations possibles. Le mot ne doit pas être contraint à vouloir signifier un seul objet bien déterminé, cela rejoint de l'arbitraire du signe. Dans Vie et Mort des Mots, Maurice Schöne avance que :

"Le mot renferme plusieurs possibilités latentes. C'est le contexte qui lui donne son sens du moment et le spécialise pour l'usage qu'en veut faire celui qui parle. ${ }^{15}$

\section{4-2 Jeux graphiques, phoniques et sémantiques}

Nous insistons pour affirmer encore une fois que le style de Boris Vian est singulier. L'effet recherché par ce dernier est une langue subversive qui sert efficacement sa pensée. C'est pour cela que l'écrivain truffe ses textes de quelques-unes de ces fantaisies graphiques et sémantiques comme la présence anarchique des accents circonflexes dans : villâge, (p.128) viû (p.47) , chûin (p.47), la présence effrénée du tréma dans les substantifs comme : fët , progrët et cröquenot (p.128)ou dans les prénoms de ces personnages Adële, Chärles, La Gloïre et Citroën entre-autres. 
Selon Gilbert Pestureau, ce "délire $d u$ tréma " ${ }^{16}$ dans les prénoms des protagonistes de n'est autre qu'une référence littéraire et un astérisque ou rappel de la célèbre marque française de voiture.

Certaines particules sont volontairement éliminées par l'auteur comme dans «béniction» (p.105) au lieu de bénidiction et dans «crobe » (p.177), considéré comme la moitié d'un microbe, «mi- » étant estimé dans cet exemple comme le suffixe et qui en représente la moitié.

Si Vian se permet certaines fantaisies orthographiques en éliminant certaines lettres, il en ajoute d'autres ailleurs, comme dans " antiquitaire » (p.241) qui est un rallongement d'antiquaire sur le modèle : société -> sociétaire.

Ne se limitant pas à la suppression ou l'ajout volontaire des lettres, Boris Vian recourt à un autre procédé qui consiste à renverser certaines formules ou parodier certaines expressions et citations connues pour les réadapter de façon personnelle et fantaisiste. Reprenant une phrase de Descartes ${ }^{17}$, il écrit :

« Jacquemort remonta, cogitant. Donc il était.» ${ }^{18}$

Nous rencontrons aussi dans L'écume des jours, une autre expression commune, une version modifiée de " suppôt de Satan ", Chez Boris, le $a$ de satin cède la place à Satan.

«Va-t’en ! criait le directeur. Suppôt de Satin !... » ${ }^{19}$

Outre les entorses graphiques et les renversements parodiques de certaines locutions, Boris Vian s'attaque à la phonétique où il use de la métathèse ${ }^{20}$ dans $L$ 'Ecume des jours, où nous rencontrons un personnage nommé Jean-Sol Partre qui renvoie bien évidemment à l'écrivain existentialiste et ami des surréalistes Jean-Paul Sartre.

Dans le même texte, quand l'auteur aborde la reliure des romans de Jean-Sol Partre, il parle du tirage qui est fait sur du "vergé Saintorix » (p.290) cette anastrophe ${ }^{21}$ est une allusion au nom du célèbre chef de la résistance gaulois Vercingétorix.

Terminons dans cette catégorie par le fameux "portecuir en feuille »(p.39). Chez Vian, on ne porte plus de billets sur soi mais plutôt le cuir qui va avec.

Jongleur des mots, amateur de farces langagières, d'autres exemples de confusion phonique occupent l'œuvre de notre auteur, le calembour ${ }^{22}$ marque sa présence dans les textes de Boris Vian. Nous relevons dans $L$ 'arrache cour :

"Le sol de carreaux usés, d'un rouge terne, jonché de petits morceaux 
de fil, de tissu, et de grains de mil pour les poules, de grains de sang pour les coqs et de grains de dix pour les amateurs. ${ }^{23}$

Dans ce passage, l'auteur emploie l'adjectif cardinal mille mais avec une graphie bien différente, celle des céréales à petits grains le mil. Il enchaine par la suite sur la même fréquence en multipliant le chiffre 10, ce qui donnera par la suite les nombres : cent et mille.

Ces deux nombres, nous les retrouvons, un peu plus loin dans la citation, sous une autre forme écrite. Cent est transformé en « sang » et dix garde sa même valeur et sémantique et graphique.

De même pour le mot " coing «, l'usage veut qu'on se retire dans un coin. Cependant, chez Vian, on se retire ailleurs, dans un fruit par exemple.

"- Non, dit Nicolas. Je voudrais me retirer dans un coing. À cause de l'odeur. Et puis parce que j'y serais tranquille... " ${ }^{24}$

Enfin, dans le même roman, nous avons le fleuve la Seine où les différents personnages risquent de s'y noyer. Avec une graphie approximative, la Seine devient une scène de «noyade ».

"Une équipe de pompiers prenaient ceux-là pour cible et, au moyen de lances d'incendie, les déviaient vers la scène où ils se noyaient misérablement.» ${ }^{25}$

Le calembour est une ressource importante du style ludique propre à Boris Vian. Se concentrant sur la sonorité semblable des différents mots, cette confusion crée une sorte de décalage entre la réalité dans laquelle nous vivons et la réalité fictive et fantastique du roman. Le calembour marque le trait humoristique de l'auteur et lui permet une approche ironique des thèmes abordés.

\section{4-3 Les discordances}

Perturbant la norme sémantique et logique à laquelle est habitué le lecteur - tout en respectant les normes grammaticales -Vian, s'amuse à créer un effet de discontinuité, de paradoxe et d'écart par rapport à la norme et à l'expérience quotidienne de celui qui le lit. Dans une cuisine pour Vian, il faut faire appel aux pompiers ou aux pompeurs.(p.311)

Une des autres possibilités de création lexicale qui permet l'invention de nouveaux mots et de nouvelles expressions est l'antithèse. Certaines formations lexicales chez l'auteur n'obéissent à aucune logique ; pourtant leur effet semble réel et sensé. 
L'écrivain d'En avant la zizique emploie des moyens rhétoriques et stylistiques classiques tel que le rapprochement de deux termes opposés comme dans le titre L'herbe rouge. En effet, dans ce roman, vers la dernière partie, l'herbe est n'est pas verte mais plutôt bien rouge voire même écarlate.

Lorsqu'on a posé la question à Mme Nicole Bertol ${ }^{\mathrm{t} 26}$ à propos de la couleur de l'herbe et pourquoi est-elle rouge ? Sa réponse était :

" Parce qu'elle n'est surtout pas verte tout simplement! ça aurait pu être l'herbe bleue... Mais avec l'herbe rouge, il y a beaucoup plus ce côté de drame, de difficulté. Le rouge c'est quand même le sang mais aussi la passion." "

Cette déclaration de la responsable de la Fond'action et du patrimoine de Boris Vian, affirme que l'auteur était un amoureux du jeu et des mots, que la couleur rouge marquait plus les esprits par la charge émotionnelle qu'elle dégage.

D'autres oxymores se dévoilent dans l'ensemble de l'œuvre, comme c'est le cas de "c'est avec une joie hargneuse... » (p.58), "L'œil droit brillait, terne...» (p.124)et «le froid de la vie... » (p.73)

Par cette juxtaposition inattendue de deux mots contraires, que tout oppose, l'auteur exprime ce qui est inconcevable, c'est-à-dire qu'il crée une nouvelle forme de réalité en juxtaposant ce qui est totalement poétique frôlant même l'absurde par moment, suscitant la curiosité du lecteur et solliciter un surplus d'activité interprétative, du fait que les deux mots de sens contraires renvoient à une même réalité.

Vian joue avec la sémantique et la phonétique des mots. Il démontre que les mots n'ont aucune limite ; qu'un mot une fois juxtaposé à son contraire peut avoir plus d'impact que s'il était associé à un terme analogue, c'est ce que l'on nomme en linguistique collocation. Employer avec audace des mots de sens opposés abolit leurs frontières. L'écart creusé avec la norme heurte la logique tout en créant un effet poétique. C'est le cas de la phrase suivante dans L'herbe rouge :

"Sexuellement, c'est-à-dire avec mon âme.» ${ }^{27}$ Cette citation relève plus de l'ordre poétique que du charnel et du physique, elle illustre mieux ce qui a été cité quelques lignes précédemment. Ici, le paradoxe ôte toute connotation érotique, habituellement attachée t sexuellement.

Nous évoquerons en dernier lieu deux figures de style : le pléonasme et l'hyperbole. Boris Vian, dans un discours prolixe, choisit des mots fantaisistes, peuple ses écrits de figures de rhétorique à redondance, de développements superflus qui frôlent l'exagération et le paroxysme. 
En effet, dans le passage qui suit, on note une volonté délibérée et d'insistance et de redondance.

«... Le tapis bleu pâle et les murs beige-rose étaient un repos pour les yeux ouverts. ${ }^{28}$

Cette redondance de détails superfétatoires permet à l'auteur d'insister sur la vérité de l'énoncé. C'est ce que Roland Barthes nomme " détails inutiles " ${ }^{29}$ dans $L$ 'effet de réel, l'écrivain ressent le besoin de laisser des traces oiseuses afin de donner un trait réel à ce qu'il avance.

De l'inutile à l'hyperbole, Vian ne manque pas d'audace langagière, comme c'est le cas de la citation suivante :

"C'était en réalité une barbe, une vaste barbe argentée qui faisait cinq à six fois le tour du corps de l'homme. " ${ }^{30}$

L'auteur fait appel à l'hyperbole qui frise d'adynaton ${ }^{31}$ qui lui sert à produire une forte impression chez le lecteur, notamment dans la description de M. Perle en le qualifiant de «Sage ». Boris Vian, pousse les limites de sa description cinq à six fois comme la barbe de son personnage.

\section{Conclusion}

Par les différentes techniques stylistiques énumérées, nous avons voulu donner un aperçu de la présentation de l'univers littéraire de l'auteur ; nous constatons qu'elles constituent les traits majeurs de son jeu verbal.

Boris Vian affiche une nette volonté de désobéissance au code langagier commun. Cette transgression et cette subversion se caractérisent par une «incivilité » vis-à-vis de la langue. Dans un effort de domination, il prend la langue à bras le corps et lui «tord le cou », la façonne, la transforme à sa guise. Ces mots glissent dans son univers, dans son imaginaire et en ressortent colorés, déformés, marqués par son empreinte et conformes à son moi intérieur. A la façon d'un compositeur de jazz qui crée une symphonie, il compose de nouveaux mots, de nouveaux sons, et sens, une nouvelle mélodie qui lui est bien singulière. 


\section{Références}

1. Paul Aron, Denis Saint-Jacques, Alain Viala, Le Dictionnaire du littéraire, Paris, PUF, 2002, p. 354.

2. «... Tu me demandes pourquoi ils ne prennent pas le livre chez Gallimard? Queneau l'aurait pris, je crois; c'est surtout Lemarchand qui ne veut pas. Je l'ai vu hier. Ils sont terribles, tous; il ne veut pas, parce qu'il me dit que je peux faire quelque chose de beaucoup mieux. C'est très gentil, mais tu te rends compte. Ils veulent me tuer, tous. Je ne peux pas leur en vouloir, je sais que c'est difficile à lire; mais c'est le fond qui leur paraît "fabriqué». C'est drôle, quand j'écris des blagues, ça a l'air sincère et quand j'écris pour de vrai, on croit que je blague...»

3. Boris Vian, Les batisseurs d'empire, Ed El'Archee impr. Laboureur et Cie (1960) p.91.

4. En linguistique c'est un mot nouveau dans une langue.

5. "Contrairement à ce qui se passe pour les mots « composés », les mots « dérivés » sont ceux dont les éléments, pourtant identifiables, ne peuvent cependant s'utiliser en dehors des formations qu'ils constituent : exclamation, préhistoire, préposition, etc. On forme un mot dérivé en adjoignant un affixe à une base déjà existante. »

6. Boris Vian, L'écume des jours, Ed, Le Livre de Poche, 2009, p.95.

7. Ibid., p. 326.

8. En linguistique : mot résultant de la fusion de deux autres mots.

9. Gilbert Pestureau, op.cit., p.146.

10. Joris- Karl Huysmans est un écrivain et critique d'art français, il a écrit en 1884 le roman À rebours.

11. Linguistique. Apparition de nouveaux sens pour un mot.

12. Boris Vian, Ibid., L'écume des jours, p., 25.

13. Boris Vian, op.cit.,p.43.

14. Boris Vian, op.cit., p, 10.

15. Schöne Maurics, Vie et Mort des Mots, Que sais-je ? P.U.F., 1959, P. 94. 
16. «Cette liste de prénoms, typique du « délire du tréma » qui emporte Vian dans ce roman, présente des références surtout littéraires : Ariel est le génie aérien de la tempête de William Shakespeare (1611), Nathanaël est «le petit pâtre» des Nourritures terrestres d'André Gide (1897), Azraël est l'ange de la mort selon la tradition de l'Islam. Pour Citroën, outre la glö̈re de cette marque de voiture dans les années 30 et 40 , due à la légendaire " Traction avant » puis, à l'autre bout de la gamme, à la « 2 chevaux», on peut penser que Vian avait été impressionné par la publicité lumineuse du nom célèbre du haut en bas de la tour Eiffel (1937). Enfin «Prünel » a un petit air germanique, sans oublier le jeu de mots sur prunelle de l'œil ou fruit du prunellier. »

17. " Je pense donc, je suis. »

18. Boris Vian, op.cit.,p.100.

19. Boris Vian, op.cit.,p.240.

20. « En linguistique, la métathèse, est une altération de l'écriture et de la prononciation d'un mot par suite de l'inversion d'une lettre ou d'un élément phonétique. EX : le mot fromage à l'origine on écrivait et on prononçait formage. »

21. Inversion; modification de l'ordre habituel des mots.

22. « Le calembour est un jeu de mots qui consiste à employer un mot dont la forme peut évoquer deux sens.(...) Souvent, le calembour exploite l'homophonie, c'est-à-dire le fait que deux mots soient identiques à l'oral mais différents à l'écrit. Le calembour consiste alors à écrire la forme qui ne convient pas dans le contexte. "

23. Boris Vian, op. cit. P, 111.

24. Boris Vian, op. cit.P, 234. 
25. Boris Vian, op. cit. P, 116.

26. Nicole Bertolt est représentante de la Cohérie Boris Vian et conseillère scientifique de l'exposition Boris Vian de la BNF.

27. Boris Vian, op.cit.,p.79.

28. Boris Vian, op.cit.,p.27.

29. Barthes Roland et al., Littérature et réalité, Paris, Seuil, 1982.

30. Boris Vian, op.cit.,p.64.

31. Un adynaton (pluriel adynata) est une figure de style qui se construit comme une hyperbole, mais dont l'effet est comique à cause de son caractère improbable. Il est assez employé dans la poésie surréaliste pour son incongruité, mais il existe aussi dans les autres formes littéraires voulant recourir à l'absurde.

\section{Références bibliographiques}

\section{Corpus}

Vian Boris, L'arrache-cœur, Paris, Ed, Le Livre de Poche, 2010.

Vian Boris , Les batisseurs d'empire, Ed El'Archee impr. Laboureur et Cie (1960)

Vian Boris, L'écume des jours, Paris,Ed, Le Livre de Poche, 2009. Vian Boris, L'herbe rouge, Paris Ed, Le Livre de Poche, 2013.

Ouvrages théoriques

Barthes Roland et al., Littérature et réalité, Paris, Seuil, 1982. Molinié, Georges Eléments de stylistique française, PUF,1986. Schöne Maurics, Vie et Mort des Mots, Que sais-je ? P.U.F., 1959. Ouvrages sur l'auteur

Pestureau Gilbert, Boris Vian, les amerlauds et les godons, Paris, Ed 1018, 1978.

Dictionnaires 
Aron Paul, Denis Saint-Jacques, Alain Viala, Le dictionnaire du littéraire, PUF, 2012. Pestureau Gilbert, Dictionnaire des personnages de Vian, Christian Bourgeois Éditeur, 1985. Pougeoise Michel, Dictionnaire didactique de la langue française, Paris, Armant Colin, 2007.

Molinié Georges, Dictionnaire de rhétorique, Le livre de Poche, Paris 2012, P,57. 